\title{
Gridded retarding pressure sensor for ion and neutral particle analysis in flowing plasmas
}

\author{
Lyon B. King a) and Alec D. Gallimore ${ }^{\text {b) }}$ \\ Plasmadynamics and Electric Propulsion Laboratory, Department of Aerospace Engineering, \\ University of Michigan, Ann Arbor, Michigan, 48105
}

(Received 2 August 1996; accepted for publication 30 October 1996)

\begin{abstract}
Although classical electrostatic plasma probes can provide detailed information regarding electrons and ions, these devices rely on charge-carrying particles both for energy filtering and for detection. Neutral particles are transparent to such diagnostics. In light of this a new probe design was developed to provide a measurement of the neutral particle flux component of a flowing plasma. The simple design adopted for this probe utilizes a set of electrostatic retarding grids to remove the charged particles from the flow. The neutral particles are sensed within the tube of an off-the-shelf hot cathode ionization gauge. Additionally, by varying the potential on the retarding grids, this same probe configuration can be used to measure the ion energy distribution. Preliminary data from this probe were taken in the exhaust plume of a Hall-current accelerator designed for space propulsion. This probe was found to be a very simple, accurate diagnostic tool for such use. By using this probe in combination with interrelated diagnostics the spatial distribution of energetic charge exchange neutrals was quantified in the plume of an SPT-100 thruster. (C) 1997 American Institute of Physics. [S0034-6748(97)01502-5]
\end{abstract}

\section{INTRODUCTION}

Electrostatic probes have widespread application in plasma diagnostics. These probes, such as Langmuir probes and retarding potential analyzers (RPAs), rely on the electric charge of ions and electrons for both energy filtering and particle detection. Neutral particles, however, are unaffected by electric or magnetic fields and are difficult to detect.

Although neutral particles are very elusive, they play an important role in many plasma processes. In electrostatic and electromagnetic space propulsion devices, for example, neutral propellant is ionized and accelerated by electromagnetic fields. Any propellant that is not ionized will therefore not be accelerated and will be unavailable for thrust. Optimizing the efficiency of such thrusters requires knowledge of the neutral propellant distribution. In addition to thruster optimization, neutral particle analysis is required to determine the exhaust plume distortion due to ground-based test facilities. ${ }^{1}$ In any ground-based vacuum facility there will exist a low density background neutral gas that is due to pump limitations. These neutral particles may collide with energetic plume particles through charge exchange collisions; such a collision produces an energetic neutral and a slow ion.

Neutral particle transport analysis can also be valuable to nuclear fusion research. Although most hot plasmas are almost completely ionized, there are neutral atoms continually being formed in the plasma interior. Because these particles travel straight across any confining magnetic field, significant numbers can escape from the plasma. These particles will carry information from the plasma concerning the state of the inner region. ${ }^{2}$

The only directly detectable properties carried by neutral particles are momentum and energy. The momentum of the

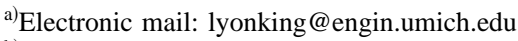

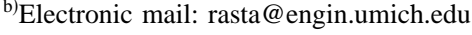

particles, represented by the particle flux, can be detected with a highly sensitive vacuum pressure gauge. However, the plasma flow of interest may have a very large ratio of charged-to-neutral particles. These charged particles also carry momentum and energy in addition to charge. The ions must therefore be filtered out by an electrostatic field prior to particle detection.

The objective of this investigation was to design, test, and evaluate a new probe capable of detecting neutral particle properties. In addition, the probe was designed to perform the dual function of an energy analyzer for energetic ions as well.

\section{DESCRIPTION OF PROBE}

The neutral particle flux probe, or NPF, is essentially a hybrid between a vacuum pressure sensor and a traditional gridded RPA. An off-the-shelf MKS Instruments tubulated hot cathode ionization gauge was used as the detector for this probe. Affixed to the inlet of the gauge tube was a set of four grids. By varying the potential on these grids particles could be selectively admitted or denied access to the tube collector. A schematic of the probe is shown in Fig. 1.

The probe body was constructed of stainless steel; the inlet aperture was $1.3 \mathrm{~cm}$ in diameter. Each of the four grid pieces was formed by sandwiching 30 line/cm electroformed nickel mesh between two 0.8 -mm-thick stainless steel washers using electrically conducting epoxy. The insulating spacers between the grid frames were constructed of $6.4-\mathrm{mm}$ thick Teflon.

The first grid and the probe body were grounded; this provided shielding from electromagnetic interference. The second grid was biased at approximately $-40 \mathrm{~V}$. This was sufficient to repel all plasma electrons from the sensing volume. The third grid was connected to a variable high voltage power supply, one capable of 0-500 V. By varying the potential of this grid, selective energy filtering of 


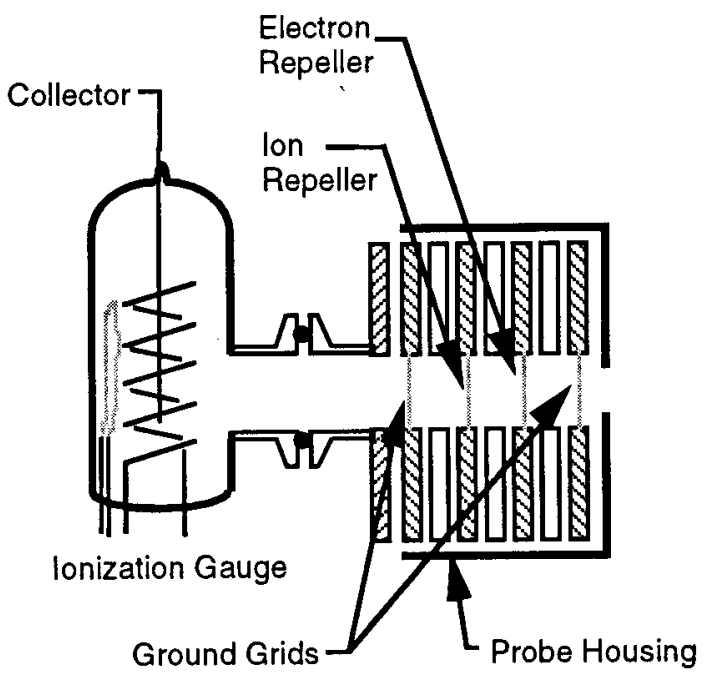

FIG. 1. Schematic of NPF probe construction.

positive ions was achieved as in a traditional RPA. By setting this ion retarding grid to the maximum positive potential all ions were prevented from entering the gauge tube. In this mode, the probe output was sensitive only to neutral particles. The fourth and final grid was grounded. This prevented any stray electric fields induced by the ion retarding grid from interfering with normal hot cathode gauge performance and calibration.

\section{DIAGNOSTIC THEORY}

The probe designed for this study was capable of measuring both neutral particle flux and ion energy distribution. The theoretical relations necessary for data reduction are now presented.

\section{A. Ion energy distribution}

The technique of using electrostatic grids to selectively retard ions is well known in plasma diagnostics. ${ }^{2}$ The NPF probe functioned as a typical RPA albeit with a nontypical current collector. Instead of using a flat surface to directly collect ion current as is typical in traditional RPA analysis, the NPF probe collected ion current along the length of the existing hot cathode gauge collector wire.

The relationship between collected current and ion retarding potential for a RPA is given as

$$
I(V)=\frac{e^{2} n_{i} A_{\text {probe }}}{m_{i}} \int_{V}^{\infty} f\left(V^{\prime}\right) d V^{\prime},
$$

where $V$ is the voltage of the ion retarding grid and $f$ is the ion energy distribution function. In Eq. (1) $A_{\text {probe }}$ is the area of the ion current collector. In traditional RPAs a planar collector is used, thus providing a clear definition of this area. For the NPF probe, this area is not well defined; the narrow collector wire of the ion gauge tube most likely does not absorb current over its entire surface area. However, this has little impact on our desired measurement. By differentiating Eq. (1) it is easily shown that

$$
-\frac{d I}{d V}=\frac{e^{2} n_{i} A_{\text {probe }}}{m_{i}} f(V) .
$$

Since we are only interested in determining the shape of $f(V),-d I / d V$ will be directly proportional to our desired function regardless of the value of $A_{\text {probe }}$.

\section{B. Neutral particle flux}

By dialing the ion retarding grid to a suitably large positive voltage all ions will be repelled from the sensing volume. In this mode the NPF probe becomes a detector for neutral particle flux.

As an output, the NPF probe measures the pressure within the ionization gauge tube. In order to glean useful information from this a relation must be derived relating the measured pressure to the free stream neutral particle flux. This can be accomplished by using free molecular theory to establish a flux balance.

At equilibrium conditions, the total flux of neutral particles entering the ionization gauge tube must be equal to the total flux of particles exiting the tube. Specifically,

$$
n_{n}\left\langle u_{n}\right\rangle_{\text {in }}=n_{m}\left\langle u_{m}\right\rangle_{\text {out }} .
$$

The flux of particles leaving the tube can be theoretically evaluated. In order to do this we assume the ionization gauge tube can be modeled as a gas reservoir containing a macroscopically stagnant equilibrium gas. Additionally, we neglect the effects of the finite length entrance to the tube and model it as an ideal orifice. If the mean free path of the gas within the tube is larger than the dimensions of the inlet orifice, then intermolecular collisions can be neglected. This is an accurate approximation: at a measured pressure of $1 \times 10^{-4}$ Torr within the tube the mean free path is greater than $10 \mathrm{~cm}$ while the inlet orifice has a diameter of $1 \mathrm{~cm}$. The escaping molecules, therefore, move through the entering molecules without interaction, and the quantities can be evaluated separately.

Denoting the positive $z$ direction exiting the orifice and assuming the gas in the ion gauge tube to be Maxwellian, the velocity distribution of molecules escaping from the ideal orifice is

$$
F_{\mathrm{esc}}=n_{m}\left(\frac{m}{2 \pi k T_{m}}\right)^{3 / 2} e^{-m\left(v_{x}^{2}+v_{y}^{2}+v_{z}^{2}\right) / 2 k T_{m}}
$$

for $v_{z}>0$, and $F_{\text {esc }}=0$ otherwise. The escape flux of particles is then integrated as

$$
\begin{aligned}
n_{m}\left\langle u_{m}\right\rangle & =\int_{-\infty}^{\infty} d v_{x} \int_{-\infty}^{\infty} d v_{y} \int_{0}^{\infty} d v_{z} v_{z} F_{\mathrm{esc}}(v) \\
& =n_{m} \sqrt{\frac{k T_{m}}{2 \pi m}}
\end{aligned}
$$

Substituting this into Eq. (3) yields an expression relating the neutral particle flux to measured quantities:

$$
n_{n}\left\langle u_{n}\right\rangle_{\text {in }}=n_{m} \sqrt{\frac{k T_{m}}{2 \pi m}} .
$$


By using the ideal gas law to relate $n_{m}$ to the measured pressure, $P_{m}$, we can close the relationship between NPF sensor output and neutral particle flux:

$$
n_{n}\left\langle u_{n}\right\rangle_{\mathrm{in}}=\frac{P_{m}}{\sqrt{2 \pi m k T_{m}}} .
$$

\section{EXPERIMENTAL PROCEDURE}

We now present a description of the experimental setup, test procedure, and parameters used in the investigation.

\section{A. Facilities}

All tests reported here were performed in the Plasmadynamics and Electric Propulsion Laboratory (PEPL) at the University of Michigan. The centerpiece of this laboratory is a large 6-m-diam by 9 -m-long space simulation vacuum chamber capable of base pressures near $2 \times 10^{-5}$ Torr. A detailed description of this facility is published elsewhere. ${ }^{3}$ All data were acquired using National Instruments signal conditioning equipment in conjunction with a computer controlled analog/digital (A/D) system.

The flowing plasma for these tests was provided by two similar devices. Both of these devices were closed drift Hall current accelerators used for electric propulsion. Extensive diagnostics were performed on a Fakel-built SPT-100 flight model thruster powered by a Space Systems/Loral power processing unit (PPU). This device has been extensively documented in literature. ${ }^{1,4-6}$ Nominal operating conditions utilized for this device consisted of $300 \mathrm{~V}$ discharge voltage at $4.5 \mathrm{~A}$. Propellant was $\mathrm{Xe}$ at $56 \mathrm{sccm}$ total flow rate with a $10 \%$ cathode split. The second device used to produce a plasma was a laboratory model of the SPT-100 built by the Moscow Aviation Institute. Nearly identical in design, the use of this laboratory model allowed adjustable settings of discharge voltage, current, and propellant flow rates.

\section{B. Setup and procedure}

In order to troubleshoot initial design iterations of the NPF probe the laboratory model thruster was used. For these tests, the NPF probe was mounted to a remote positioning system within the vacuum chamber (described in Ref. 3) and placed at various locations within the thruster plume. Aspects such as cable design, EMI minimization, and thruster thermal impacts were investigated in these tests.

Once the operational difficulties with the probe had been corrected, a comprehensive suite of diagnostics was performed using the NPF and other probes on the flight model SPT-100; the results of this investigation are presented elsewhere. ${ }^{1}$ For these investigations plume surveys of the SPT-100 were taken at a $0.5 \mathrm{~m}$ radial distance from the thruster exit plane as well as at $1.0 \mathrm{~m}$.

\section{Probe operation}

The hot cathode ionization gauge sensor on the NPF was controlled using a standard MKS model 919 controller. The 919 was calibrated for Xe in units of Torr. Both ion and electron retarding potentials were provided by laboratory power supplies.
During operation of the NPF in neutral flux mode the 919 controller required no modifications. However, some difficulties were encountered during operation in RPA mode; these will be described later in Sec. V. These difficulties required that the ionization gauge grid and filament be deactivated while still monitoring the current from the collector. Through factory consultation this was accomplished by simply repositioning three internal dip switches within the controller.

\section{RESULTS}

\section{A. Ionization gauge verification}

In order to allow remote positioning of the NPF probe within the volume of the vacuum chamber a custom set of power cables was constructed. These cables, fed through the vacuum chamber wall, had an overall length of approximately $20 \mathrm{~m}$. The NPF probe ionization gauge operation was verified by simply comparing the pressure measured at stagnant vacuum (no plasma flow) to the pressure measured by existing ionization gauges on the tank. During this initial verification it was discovered that the NPF probe ionization gauge indicated a measured pressure of around $3 \times 10^{-7}$ Torr whereas the tank pressure was actually at $4 \times 10^{-5}$ Torr. The cause of this was identified as signal power loss in the lengthy cables. This problem was remedied by shortening the cables to approximately $10 \mathrm{~m}$. After this configuration change the NPF ionization gauge confirmed the pressure measured by the tank gauges. Pressure measured by the ionization gauge showed absolutely no change when voltage was applied to either the ion or electron retarding grids in the absence of plasma.

\section{B. RPA mode}

The ability of the NPF sensor to measure the ion energy distribution function was tested by operating the sensor in RPA mode. This testing was performed in the plume of the laboratory model Hall thruster.

The electron retarding grid was set to a potential of -40 $\mathrm{V}$; this value was suitable to repel all plasma electrons in this flow. ${ }^{7}$ By adjusting the potential of the ion retarding grid ions were permitted to enter the ionization gauge tube according to Eq. (2). The 919 controller converted the current measured by the ionization gauge collector wire into a proportional equivalent pressure in units of Torr. Initial tests revealed an interesting complication due to the ionization gauge. The data from this RPA-type trace of pressure (current) versus ion retarding potential is shown as Fig. 2. It should be noted that the preliminary data represented in Fig. 2 were obtained with the $20 \mathrm{~m}$ probe cables. As stated earlier, the erroneously low pressures indicated in Fig. 2 were due to signal loss in the cables.

The behavior of the data in Fig. 2 is due to the field configuration within the ionization gauge tube. The ionization gauge tube senses pressure (usually due to neutral particles) by ionizing a fraction of the particles through electron impact ionization, then sensing the ion current with the collector. The collector is encircled by a helical grid that is used to collect electrons. In order to ensure efficient ion current 


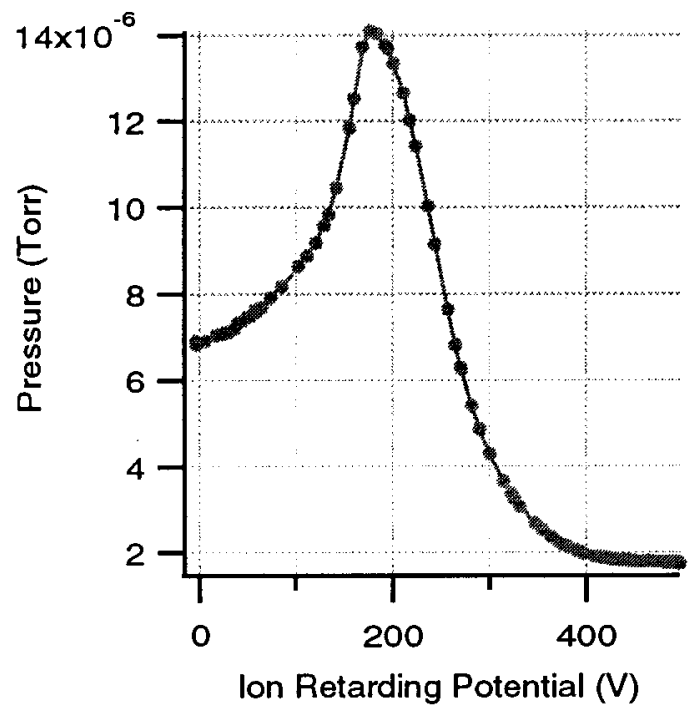

FIG. 2. NPF sensor output in RPA mode showing effect of ionization gauge grid bias voltage. Laboratory thruster used with $V_{d}=200 \mathrm{~V}, I_{d}=4.2 \mathrm{~A}$. Probe $15^{\circ}$ off thruster centerline $73 \mathrm{~cm}$ radius from exit plane.

collection the gauge grid voltage is maintained at $180 \mathrm{~V}$ while the collector is held at ground. This causes the collector to have the lowest potential. When the ionization gauge tube is exposed to an incident flow of plume ions in addition to the tube-formed ions, the interaction of the gauge grid voltage with the NPF ion retarding grid causes the peak shown in Fig. 2. This can be understood by examining Fig. 3.

When the ion retarding grid has a voltage of less than $180 \mathrm{~V}$, it has no effect on the incident plume ions that are capable of reaching the collector; the ion gauge grid voltage repels all ions with energies of less than $180 \mathrm{~V}$ from direct impingement on the collector. The ion retarding grid does, however, have an effect on the measured pressure due to its

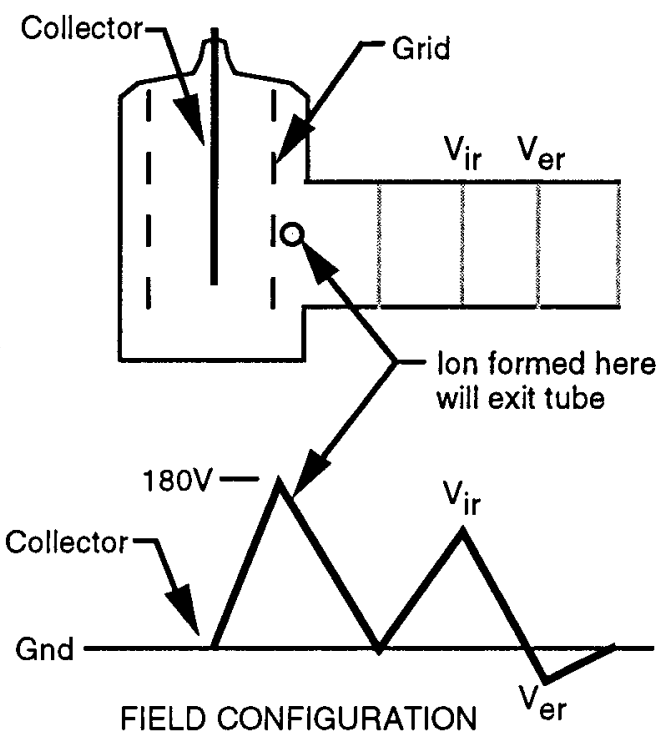

FIG. 3. Schematic showing interaction of electric fields in ionization gauge with the NPF grids.

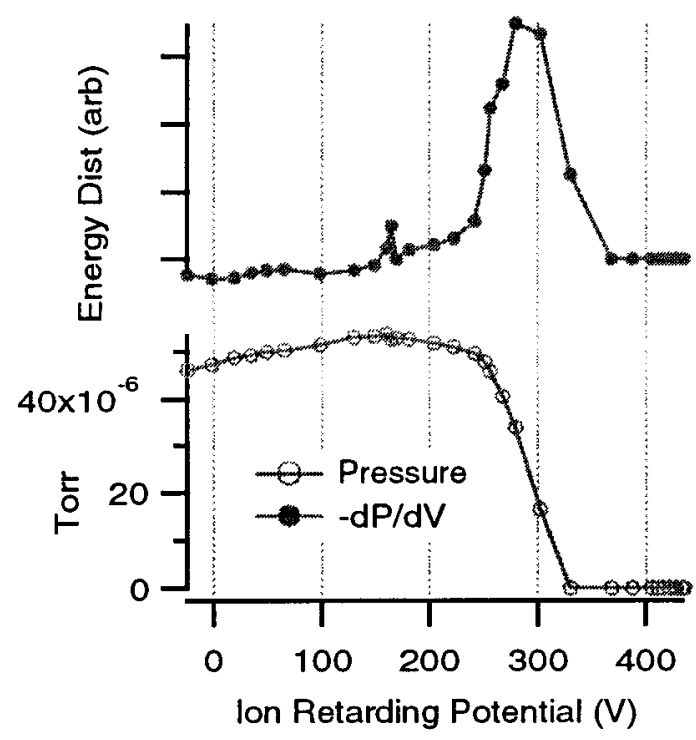

FIG. 4. A RPA mode trace taken with the ionization gauge grid and filament deactivated at $0.5 \mathrm{~m}$ radius from the SPT-100 $15^{\circ}$ off thruster centerline.

interaction with ions formed within the tube due to electron impact ionization. Some ions formed immediately outside of the gauge grid will have sufficient energy to overcome the ion retarding grid field and will exit the tube undetected. However, some ions formed outside of the gauge grid will not have enough energy to overcome this barrier. These ions will be reflected back into the tube. This will effectively increase the density and hence the measured pressure within the tube. At an ion retarding potential of $180 \mathrm{~V}$, no ions formed within the tube will be capable of exiting the tube and hence the measured pressure will be a maximum. As the potential of the ion retarding grid is raised above $180 \mathrm{~V}$, the number of plume ions capable of entering the gauge will be reduced as in a traditional RPA, and the measured pressure will again decrease.

The sensor, then, operates like a "two way" RPA due to the interaction of the fields. The portion of the curve in Fig. 2 below $180 \mathrm{~V}$ represents a RPA characteristic for ions formed within the tube, while the portion of the curve above $180 \mathrm{~V}$ represents a RPA characteristic for plume ions.

In order to eliminate this adverse field interaction it was necessary to deactivate the filament and grid mechanisms within the ionization gauge tube during operation of the NPF in RPA mode. Subsequent to manufacturer consultation, this was accomplished by repositioning three dip switches within the 919 controller. The controller, now reconfigured, simply operated as a high resolution ammeter monitoring the ion current incident on the collector wire. The results of a RPA trace taken in the plume of the flight model SPT with corrected $10 \mathrm{~m}$ cables and deactivated gauge grid and filament are shown in Fig. 4.

\section{Neutral flux model}

By analyzing the data shown in Fig. 4 it was apparent that an ion retarding voltage of $500 \mathrm{~V}$ was sufficient to repel all plume ions from entering the ionization gauge tube. In 


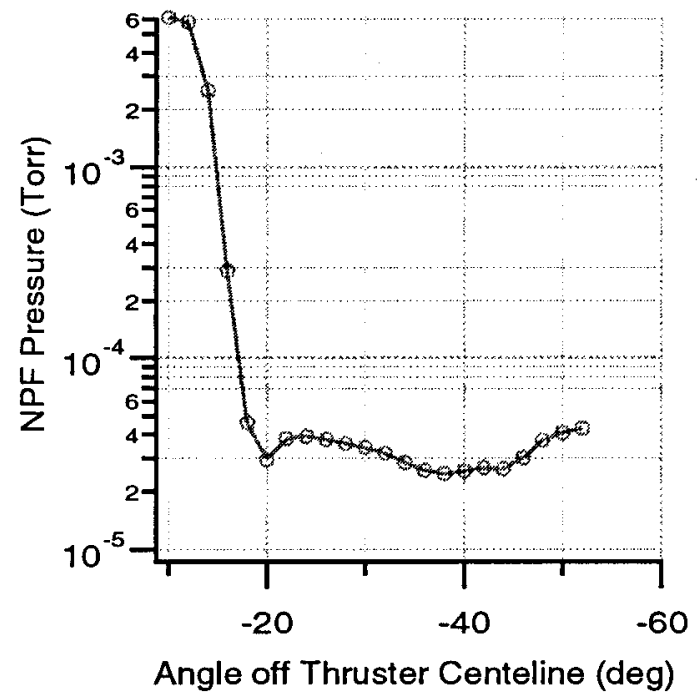

FIG. 5. NPF sensor output in neutral flux mode at $0.5 \mathrm{~m}$ radius from the SPT-100 exit plane.

this mode of operation the ionization gauge filament and grid were activated as per normal operation of the tube, and the pressure indicated by the 919 controller was due entirely to neutral particles entering the tube volume.

Extensive data were taken in neutral flux mode in the plume of the flight model SPT-100. This data is detailed in Ref. 1 and will be summarized here.

At a radial position of $0.5 \mathrm{~m}$ from thruster exit plane the sensor was rotated from thruster centerline through $60^{\circ}$ off centerline in $2^{\circ}$ increments. The sensor output is shown as Fig. 5.

In order to prevent distruction of the ionization gauge filament, the 919 controller utilizes an overpressure protect setpoint. Above this pressure the gauge tube is deactivated. Although the setpoint for this function was increased to the maximum pressure permitted by the controller, the ionization gauge still exceeded this pressure for points within $10^{\circ}$ of thruster centerline. Therefore data in this regime were not obtained.

By using Eq. (6), the raw output of the NPF sensor can be converted to units of neutral particle flux. In order to verify this data a separate analysis of neutral particle properties was used as a comparison. By combining measurements of convective heat flux due to both ions and neutrals with convective heat flux due only to ions it was possible to calculate the convective heat flux due to the neutral particles only. ${ }^{1}$ This neutral convective heat flux is proportional to the third moment of the neutral particle velocity distribution function whereas the NPF-measured flux is proportional to the first moment. A comparison between these two quantities is shown as Fig. 6.

Both the NPF probe data and the calculated neutral particle heating show almost identical trends. This suggests that the NPF probe-measured neutral particle flux is an accurate physical diagnostic.

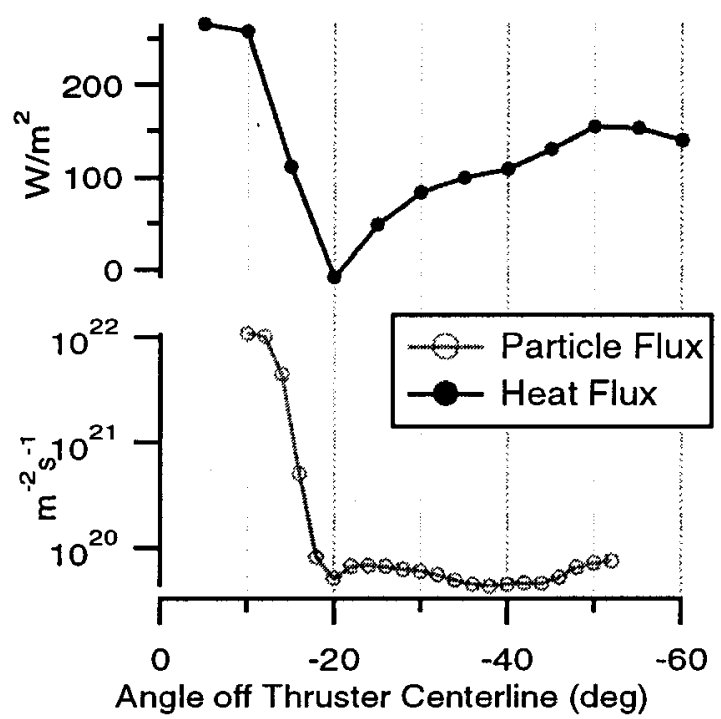

FIG. 6. Comparison between NPF measured neutral flux and calculated neutral particle convective heat flux at $0.5 \mathrm{~m}$ radius from the exit plane of the SPT-100.

\section{DISCUSSION}

Once the complication due to the ionization gauge grid voltage was identified and corrected, the pressure (current) versus voltage trace obtained was typical of a traditional RPA. The trace obtained as Fig. 3 is very comparable to RPA data obtained with a proven conventional RPA in parallel investigations. ${ }^{1}$ For this preliminary probe design the measured ion energy distribution agrees well with the accepted form for this thruster. However, for future design iterations of this probe one improvement would be beneficial: currently the probe body and first grid are grounded. This imposes a disturbance to the flowing plasma, whose potential may be several volts above tank ground. It would be advantageous to allow the probe body and first grid to float. This would minimize the plasma disturbance and reduce the uncertainty in the ion energy distribution function.

By comparing the NPF measured neutral particle flux to calculated values of neutral particle heating it is apparent that the trends are nearly identical. This agreement between separate diagnostic techniques strongly suggests that the NPF sensor output reflects true physical quantities. Although an accurate absolute calibration of the sensor was not performed, a simple free molecular analysis can be used to provide useful quantitative data.

The NPF probe measurements of neutral particle flux showed great sensitivity, however the range of the ionization gauge sensor proved too small for the plasma flows investigated in this study. For points within $10^{\circ}$ of thruster centerline at $0.5 \mathrm{~m}$ radius the tube pressure induced by the neutral particle flow was higher than the maximum range of the ionization gauge. This caused a region of unobtainable data. Future versions of this probe technique will also employ an extended range ionization gauge tube that is widely available from commercial vacuum vendors. Such tubes have allowable maximum pressures up to $50 \mathrm{mTorr}$; this would avoid the out-of-range scenario encountered in this investigation. 


\section{NOMENCLATURE}

$A_{\text {probe }}=$ area of RPA current collector $\left(\mathrm{m}^{2}\right)$

$e=$ elementary charge (coulomb)

$F_{\text {esc }} \quad=$ escaping particle velocity distribution

$f \quad=$ ion energy distribution function

$I \quad=$ collected current $(\mathrm{A})$

$k \quad=$ Boltzmann constant

$m_{i} \quad=$ mass of ion $(\mathrm{kg})$

$n_{i} \quad=$ density of ions $\left(\mathrm{m}^{-3}\right)$

$n_{n} \quad=$ density of neutrals $\left(\mathrm{m}^{-3}\right)$

$n_{m} \quad=$ measured density $\left(\mathrm{m}^{-3}\right)$

$P_{m} \quad=$ measured pressure $(\mathrm{Pa})$

$T_{m} \quad=$ measured temperature $(\mathrm{K})$

$u_{n} \quad=$ velocity of neutrals $(\mathrm{m} / \mathrm{s})$

$u_{m} \quad=$ measured particle velocity $(\mathrm{m} / \mathrm{s})$

$V \quad=$ ion retarding voltage $(\mathrm{V})$

$\langle x\rangle \quad=$ average value of quantity " $x$ "

\section{ACKNOWLEDGMENTS}

The research reported herein was sponsored by the Air Force Office of Scientific Research; this support is gratefully acknowledged. In addition, the authors would like to thank Mike Day for loan of SPT-100 and PPU, machinist Terry Larrow, and PEPL researchers Matt Domonkos, John Foster, James Haas, Sang-Wook Kim, and Colleen Marrese.

${ }^{1}$ L. B. King and A. D. Gallimore, 32nd AIAA/ASME/SAE/ASEE Joint Propulsion Conference, 1-3 July 1996, Orlando, FL, paper no. AIAA-962712.

${ }^{2}$ I. H. Hutchinson, Principles of Plasma Diagnostics (Cambridge University Press, New York, 1987).

${ }^{3}$ A. D. Gallimore, S. W. Kim, J. E. Foster, L. B. King, and F. S. Gulczinski III, AIAA J. Prop. Power, 12, 105 (1996).

${ }^{4}$ C. M. Marrese, A. D. Gallimore, J. Haas, J. E. Foster, L. B. King, and S. W. Kim, 31st AIAA/ASME/SAE/ASEE Joint Propulsion Conference, 10-12 July 1995, San Diego, CA, paper no. AIAA-95-2932.

${ }^{5}$ A. M. Bishaev and V. Kim, Sov. Phys. Tech. Phys. 23, 1055-1057 (1978).

${ }^{6}$ D. H. Manzella, 30th AIAA/ASME/SAE/ASEE Joint Propulsion Conference, 27-29 June 1994, Indianapolis, IN, paper no. AIAA-94-3141.

${ }^{7}$ R. M. Myers and D. H. Manzella, 23rd International Electric Propulsion Conference, Sept. 1993, Seattle, WA, paper no. IEPC-93-096. 